\title{
The CdZnTe Detector with Slit Collimator for Measure Distribution of the Specific Activity Radionuclide in the Ground
}

\author{
V.E. Stepanov, A.G. Volkovich, V.N. Potapov, I.A. Semin, A.V.Stepanov, Iu.N. Simirskii \\ National Research Center "Kurchatov Institute" - Moscow (Russian Federation)
}

\begin{abstract}
From 2011 in the NRC "Kurchatov Institute" carry out the dismantling of the MR multiloop research reactor. Now the reactor and all technological equipment in the premises of the reactor were dismantled. Now the measurements of radioactive contamination in the reactor premises are made. The most contaminated parts of premises - floor and the ground beneath it. To measure the distribution of specific activity in the ground the CdZnTe detector (volume 500мм3) was used. Detector placed in a lead shielding with a slit collimation hole. The upper part of shielding is made movable to close and open the slit of the collimator. At each point two measurements carried out: with open and closed collimator. The software for determination specific activity of radionuclides in ground was developed. The mathematical model of spectrometric system based on the Monte-Carlo method. Measurements of specific activity of ground were made. Using the results of measurements the thickness of the removed layer of ground and the amount of radioactive waste were calculated.
\end{abstract}

\section{INTRODUCTION}

From 2011, specialists of the NRC Kurchatov institute carry out the dismantling of MR and RFT research reactors. The RFT 20 MW water-graphite materials-research channel reactor, including the loop facilities, was commissioned in 1952. The reactor consisted of five loop setups for testing fuel assembly and structural materials. After intense operation for 10 years, it was stopped in 1962 and partially dismantled. In the same hall was built a more powerful multiple-loop reactor MP.

The MP reactor was a multipurpose material research nuclear reactor with 9 loops setups. These loops provided an opportunity to model the operating conditions for various types of fuel assemblies from different nuclear power plants, and were equipped with appropriate elements of contour equipment. A pond with reactor internals, a pond for spent fuel assemblies, technological equipment of reactor and loop setups, equipment of first and second cooling circuit pipelines are in the basement rooms of the reactor building. Operating condition of the reactor and its loop setups during the 30 -year period of time increased radioactive contamination of equipment, reactor cooling pipelines system, and loops setups.

The reactor MR was finally shutdown in December 1992. In 1996 the fuel was removed from active zone ant reactor was transferred to a nuclear-safe state. In 2013-2014 all loops setup and equipment from 47 basement rooms were dismantled. Dismantling of equipment allowed to reduce the exposure dose rate (EDR) in premises from $5-20 \mathrm{mSv} / \mathrm{h}$ to $0.01-0.03 \mathrm{mSv} / \mathrm{h}$. The dismantling of reactor internal constructions was started in 2014. In 2015 the vessel of the MR reactor and the graphite stack of the RFT reactor were dismantled [1-6].

In 2016 the measuring of contamination in basements rooms and removing the radioactive waste (soil, brick and concrete rubble) were begun. The most contaminated parts of the premises are the floor (concrete) and the ground under it. This radioactive contamination is highly inhomogeneous by the area of premises and by the depth of penetration into the ground.

The area under the reactor is about $1300 \mathrm{~m}^{2}$. If we assume that the thickness of the contaminated layer is, for example, $0.2 \mathrm{~m}$, the volume of radioactive waste will be $\sim 260 \mathrm{~m}^{3}$. Measurement of detailed distribution of specific activity of radionuclides in soil by the area and by depth will allow significantly reduce the amount of the radioactive waste. To measure the distribution of specific activity in ground the collimated spectrometric detector was developed.

\section{Collimated Spectrometric Detector}

The scheme of the spectrometric collimated detector (SCD) is shown in fig.1. To measure the distribution of specific activity in the soil, the CdZnTe detector (volume $500 \mathrm{~mm}^{3}$ ) is used (3). The detector is placed in a lead shield (2.4) with a slit collimation hole. Lead shield is located inside the steel case of the device (1). The upper part of the lead shield is movable (5rod for moving the upper part of the shield) and provide to close and open the collimator slit. Two measurements are carried out at each point: with an open and closed collimator. The energy resolution of the detector at the $662 \mathrm{keV}$ line is 7.2 $\mathrm{keV}$. The dimensions of the detector CdZnTe: $\varnothing 24 \mathrm{~mm}, \mathrm{~L}=$ $58 \mathrm{~mm}$. 


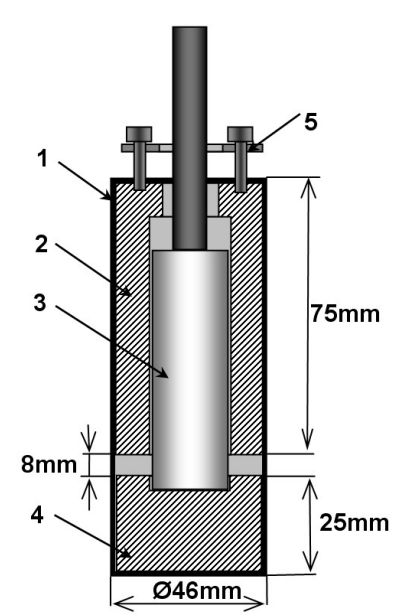

Fig.1. The scheme of collimated spectrometric detector (1-case; 2,4- lead shield; 3 -detector; 5 - movable rod).

SCD move along the hole in the ground with discrete steps and make two measurements in each point. After carrying out measurements, the values of the specific activity of a soil are calculated in specially developed software.

\section{III.METHOD FOR EVALUATED SPECIFIC ACTIVITY OF SOIL}

We assume that a specific activity of ${ }^{137} \mathrm{Cs}\left(\mathrm{A}_{\mathrm{Cs}}\right)$ has a uniform distribution in a soil by the depth of the well. The counting rate in the peak of total absorption is formed only by the unscattered emission of ${ }^{137} \mathrm{Cs}$. The count rate is related to the specific activity by a simple equation:

$$
n_{C s}=\frac{S \varepsilon n_{\gamma} \vartheta_{\max }}{2 \mu\left(E_{0}\right)} A_{C s}
$$

Where $\eta_{\gamma}$ is the yield of $\gamma$-quanta ${ }^{137} \mathrm{Cs}$ on one decay, $S$ - the effective cross-sectional area of the detector crystal, $\varepsilon-$ the detection efficiency of the unscattered emission of ${ }^{137} \mathrm{Cs}$, $\vartheta_{\max }-$ the effective angle of the detector collimator, $\mu\left(E_{0}\right)$ the mass attenuation coefficient in medium of the $\gamma$-emission with $E_{0}$ energy (in this case $E_{0}=662 \mathrm{KeV}$ ).

From the equation (1) follows:

$$
n_{C s}=C \times A_{C s}
$$

Here $C=\frac{S \varepsilon n_{\gamma} \vartheta_{\max }}{2 \mu\left(E_{0}\right)}$ is a coefficient of proportionality.

The coefficient of proportionality is equal to the sensitivity of the detector and determined by calibrating the spectrometric collimated detector. Thus, from equation (2) follows, that the specific activity of ${ }^{137} \mathrm{Cs}$ can be determined by the formula:

$$
A_{C s}=n_{C s} \times C^{-1} \text {, }
$$

$\mathrm{C}$ - is the calibration factor, which is equal to the counting rate in the peak of the total absorption of the $\gamma$ - emission of ${ }^{137} \mathrm{Cs}$ in a medium with a unit specific activity concentration.
According to the equation (2), the calibration factor is universal, i.e. it practically does not depend on chemical composition of the environment, since the mass attenuation coefficient (soil or concrete) for $\gamma$-quanta energies from 0.5 to $2 \mathrm{MeV}$ weakly depends on atom number of chemical elements in investigated substance.

The relative error of the measured activity will be determined by the relative error of the counting rate, $\eta_{c s}$ i.e.

$$
\delta_{A}=\frac{\Delta A_{C s}}{A_{C s}}=\frac{\Delta n_{C s}}{n_{C s}}
$$

The statistical error $\Delta \eta_{C s}$, is determined by formula (2). The similar method and the same equations can be used for the other radionuclides specific activity evaluation.

The spatial resolution of the SCD depends on the angle of collimation $\vartheta_{\max }$. Increasing the spatial resolution of the detector (decreasing $\vartheta_{\max }$ ) leads to sensitivity decrease. Therefore, the optimal choice of the collimator parameters is a compromise between the sensitivity and the spatial resolution. The spatial apparatus feature for the developed spectrometric collimated detector with the geometric parameters shown in fig. 1 was calculated (fig.2).

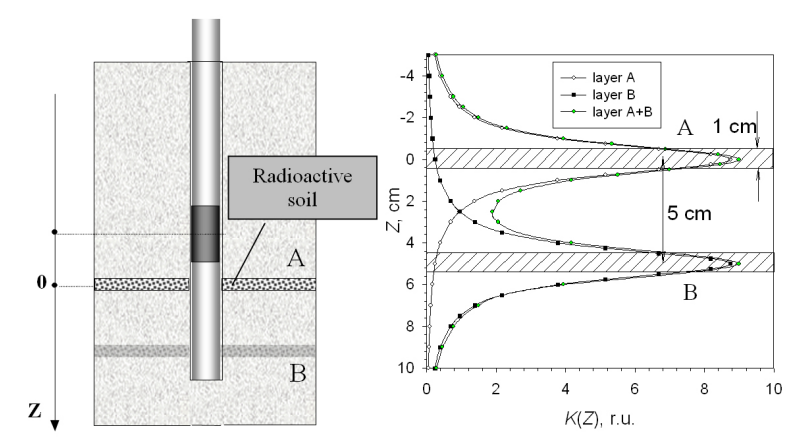

Fig. 2. The spatial hardware feature of the spectrometric collimated detector (A and B layers $1 \mathrm{~cm}$ thickness are located at a distance of $5 \mathrm{~cm}$ from each other, with the uniform distribution ${ }^{137} \mathrm{Cs}$ activities).

The spatial hardware feature of the spectrometric collimated detector is the dependence of the counting rate in the peak of total absorption on the position of the detector (coordinate $\mathrm{Z}$ ) relative to the layer of soil (1 cm thickness) with the ${ }^{137} \mathrm{Cs}$ radionuclide activity uniform distributed in it. Two layers with $1 \mathrm{~cm}$ thickness (layer A and B) at a distance of $5 \mathrm{~cm}$ from each other are shown in left part of fig.2. Dependence of the counting rate on moving of the detector along the $\mathrm{Z}$ direction (perpendicular to the layers) is shown in right part of fig.2. From this figure it can be seen that the spatial resolution of the detector with such geometric parameters of the collimator makes it possible to detect two separate thin layers of contaminated soil. This indicates that the spatial resolution of such a spectrometric system is about $3 \div 4 \mathrm{~cm}$.

\section{Collibration of Collimated SPectrometric DETECTOR}


To carry out the calibration, a point source with activity $A_{C s}^{\text {point }}$ was placed at a distance $L$ from the detector and the counting rate at the peak of the total absorption $n_{C s}^{p o \text { int }}$ is determined. Then, using the mathematical model of this detector, two calculations are performed: the counting rate $n_{C s}^{\text {calc }}$ for a point source with the same activity and the counting rate $\tilde{n}_{C s}^{\text {calc }}$ for a uniformly distributed source in a medium with a specific activity of $1 \mathrm{~Bq} / \mathrm{kg}$. The assumed count rate of the peak of the total absorption of the SCD for a uniform distributed source will be determined by the formula:

$$
\widetilde{n}_{C s}=\widetilde{n}_{C s}^{\text {calc }} \cdot \frac{n_{C s}^{\text {point }}}{n_{C s}^{\text {calc }}}
$$

This count rate determines the calibration coefficient between the soil specific activity and the count rate in a peak of total absorption of the ${ }^{137} \mathrm{Cs}$. The sensitivity of the detector in the peak total absorption at the $662 \mathrm{keV}$ line $\left({ }^{137} \mathrm{Cs}\right)$ was calculated as $1.74 \mathrm{KBq} \cdot \mathrm{kg}^{-1} \cdot \mathrm{s}^{-1}$. The same method was used to determine calibration coefficients for other radionuclides. This procedure is described in more detail in work [7].

\section{MEASURMENTS OF RADIOACTIVE SOIL CONTAMINATION}

Exposure dose rate in reactor basements rooms is mainly determined by radiation from the walls and floor. The main $\gamma$ emitting radionuclides are ${ }^{137} \mathrm{Cs}$ and ${ }^{60} \mathrm{Co}$. Before the removal of contaminated soil from the reactor premises, a comprehensive radiation survey was carried out. This survey consisted of:

- EDR distribution measurements in all rooms;

- measurements of radioactive contamination of the surfaces by the $\beta$-detector;

- measurements of distribution of a specific activity in soil by the collimated detector [8];

- drilling of technological holes and measuring distributions of EDR in it;

- measurement of a specific activity of a soil along depth by means of spectrometric collimated detector.

The technological holes were drilled in is most radioactive contaminated areas of the rooms. The SCD was moving with a discrete step along the hole. Two measurements (with an open and closed collimator) were carried out at each point of the profile. The resultant spectrum (a difference between spectra with the opened and closed collimator) at a depth of $15 \mathrm{~cm}$ in one of the hole is shown in fig.3.



Fig.3. The resultant spectrum (a difference between spectra with the opened and closed collimator)
The distribution of the soil specific activity and the distribution of EDR along the depth of the technological hole in the room №64 are shown in fig.4.

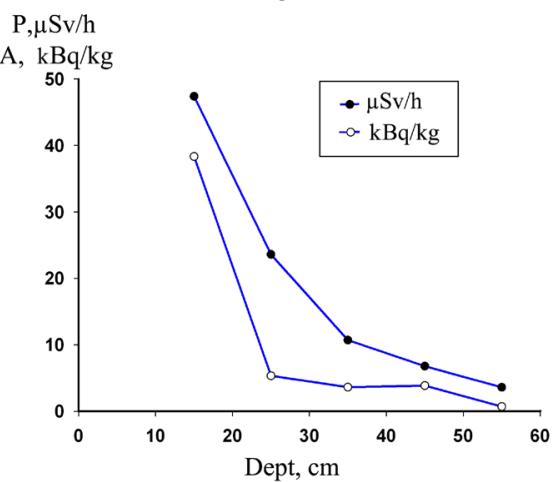

Fig.4. The distribution of the soil specific activity and the distribution of EDR along the depth of the technological hole.

The graph shows, that the change of EDR values along the depth of the hole is more smooth than the change the values of specific activity. This is explained by the fact that the contribution to the EDR at each point gives a greater amount of soil having different specific activity. Measurements made with the spectrometric collimated detector give more detailed information about the distribution of specific activity along the depth in the hole. The results of the measurements with SCD were compared with the results of laboratory measurements of soil samples. These measurements were estimated by the spectrometric complex InSpector-2000 of the Canberra Company that included a semiconductor detector using HP Germanium GC-4018. The analysis of the $\gamma$-spectrum was made by the GENIE-2000 software. The value of the specific activity of the soil sample taken from a depth of $15 \mathrm{~cm}$ from the hole in room № 64 was $-23 \mathrm{kBq} / \mathrm{kg}$, the SCD measurement at the same depth was $18 \mathrm{kBq} / \mathrm{kg}$. This result shows a good agreement, despite the fact that the measurements by sample method are much less representative compared to measurements by a SCD.

\section{CONCLUSION}

The radiation survey of the reactor basement rooms showed that the radioactive contamination is highly non-uniformed by area and by the depth. Using SCD for measuring the distributions of specific activity in the soil by the depth in the reactor premises showed high efficiency. Based on this measurements the sorting of the soil according to specific activity and estimating the volume of removing ground were made. The most contaminated soils, in accordance with the regulations rules, were packed in containers for disposal. Part of the soil was sent to the soil cleaning unit [9], after which it returned to the backfill.

\section{REFERENCES}

[1] V.G. Volkov, Y.A. Zverkov etc., "Preparation for the decommissioning of research reactor MR in the Kurchatov Institute", Atomic Energy, vol.104, № 5, pp. 259-264, 2008 
[2] A.G. Volkovich, O.P. Ivanov, A.V. Lemus etc., "Particularities of the Dismantling of the Intra-Vessel Structures of the RFT Reactor”, Atomic Energy, vol.121, № 5, pp. 377-382, 2017

[3] Alexander Chesnokov, Oleg Ivanov, Vyacheslav Kolyadin et al. "HLRW management during MR reactor decommissioning in NRC "Kurchanjv insninute", ICEM'13 Conference Proceedings - 96046, Brussels, Belgium, September 8-12, 2013.

[4] Stepanov V.E., Ivanov O.P. et al., "Remote-controlled collimated $\gamma$-ray detector for measuring radioactive contamination”, Atomic energy, vol.109, №2, pp. 102105,2010

[5] Ivanov O.P., Danilovich A.S., Stepanov V. E. et al., "Experience of application of new remote controlled instruments for scanning of distribution of radioactive contamination in rooms with high dose rate", ICEM'11 Conference Proceedings - 59354, Reims, France, September $25-29,2011$

[6] Alexey Danilovich, Oleg Ivanov, Alexey Lemus, Sergey Smirnov, Vyacheslav Stepanov et al., "Radiological survey of contaminated installations of research reactor before dismantling in high dose conditions with complex for remote measurements of radioactivity." WM2012 Conference Proceedings - 12069, Phoenix, Arizona, USA, February 26 March 1, 2012

[7] Vyacheslav E.Stepanov, Victor N.Potapov, Sergey V.Smirnov, Oleg P.Ivanov. "Characterizations of the Radioactive Waste by the Remotely-Controlled Collimated Spectrometric System". ANIMMA2015, Book of Proceedings -IQ-45, Lisboa, Portugal, April 20 - 24, 2015.

[8] Alexey Stepanov,Oleg Ivanov, Victor Potapov et al. "Development and Application of Collimated Spectrometric Systems for the Characterization of Radioactive Contamination of Decommissioned Facilities", WM2015 Conference Proceedings - 15030, Phoenix, Arizona, USA, March 1, 2015

[9] Victor Volkov, Alexander Chesnokov, Alexey Danilovich et al., "Techniques of radioactive soil processing at rehabilitation of contamination territories", ICEM'11 Conference Proceedings - 59199, Reims, France, September $25-29,2011$ 